\title{
Gaia's Cepheids and RR Lyrae stars and luminosity calibrations based on Tycho-Gaia Astrometric Solution
}

\author{
Gisella Clementini $^{1, \star}$, Laurent Eyer ${ }^{2}$, Tatiana Muraveva ${ }^{1}$, Alessia Garofalo ${ }^{1,3}$, Vincenzo Ripepi ${ }^{4}$, \\ Marcella Marconi ${ }^{4}$, Luis Sarro ${ }^{5}$, Max Palmer ${ }^{6}$, Xavier Luri ${ }^{6}$, Roberto Molinaro ${ }^{4}$, Lorenzo \\ Rimoldini ${ }^{7}$, Laszlo Szabados ${ }^{8}$, Richard I. Anderson ${ }^{9}$, and Ilaria Musella ${ }^{4}$ \\ ${ }^{1}$ INAF - Osservatorio Astronomico di Bologna, Via Gobetti 93/3, 40129 Bologna, Italy \\ ${ }^{2}$ Department of Astronomy, University of Geneva, Ch. des Maillettes 51, 1290 Versoix, Switzerland \\ ${ }^{3}$ Dipartimento di Fisica e Astronomia, Università di Bologna, Via Gobetti 93/2, 40129 Bologna, Italy \\ ${ }^{4}$ INAF-Osservatorio Astronomico di Capodimonte, Via Moiariello 16, 80131 Napoli, Italy \\ ${ }^{5}$ Dpto. Inteligencia Artificial, UNED, c/ Juan del Rosal 16, 28040 Madrid, Spain \\ ${ }^{6}$ Institut de Ciències del Cosmos, Universitat de Barcelona (IEEC-UB), Martí Franquès 1, E-08028 \\ Barcelona, Spain \\ ${ }^{7}$ Department of Astronomy, University of Geneva, Ch. d'Ecogia 16, 1290 Versoix, Switzerland \\ ${ }^{8}$ Konkoly Observatory, Research Centre for Astronomy and Earth Sciences, Hungarian Academy of Sci- \\ ences, Konkoly Thege Miklós út 15-17, 1121 Budapest, Hungary \\ ${ }^{9}$ Department of Physics and Astronomy, The Johns Hopkins University, $3400 \mathrm{~N}$ Charles St, Baltimore, MD \\ 21218, USA
}

\begin{abstract}
Gaia Data Release 1 contains parallaxes for more than 700 Galactic Cepheids and RR Lyrae stars, computed as part of the Tycho-Gaia Astrometric Solution (TGAS). We have used TGAS parallaxes, along with literature $\left(V, I, J, K_{\mathrm{s}}, W_{1}\right)$ photometry and spectroscopy, to calibrate the zero point of the period-luminosity and period-Wesenheit relations of classical and type II Cepheids, and the near-infrared period-luminosity, period-luminosity-metallicity and optical luminosity-metallicity relations of RR Lyrae stars. In this contribution we briefly summarise results obtained by fitting these basic relations adopting different techniques that operate either in parallax or distance (absolute magnitude) space.
\end{abstract}

\section{Introduction}

Cepheids and RR Lyrae stars are primary standard candles of the cosmological distance ladder and excellent tracers of young (the classical Cepheids) and old (the RR Lyrae stars and the Type II Cepheids) stellar populations. Gaia will be an extraordinary discoverer of new Cepheids and RR Lyrae stars in and beyond the Milky Way. Furthermore, the unprecedented accuracy of Gaia's end-of-mission astrometric measurements will allow us to pin down the slope and zero point of the fundamental relations that Cepheids and RR Lyrae stars conform to with the precision required to constrain the Hubble constant to better than $2 \%$.

${ }^{\star}$ gisella.clementini@oabo.inaf.it 


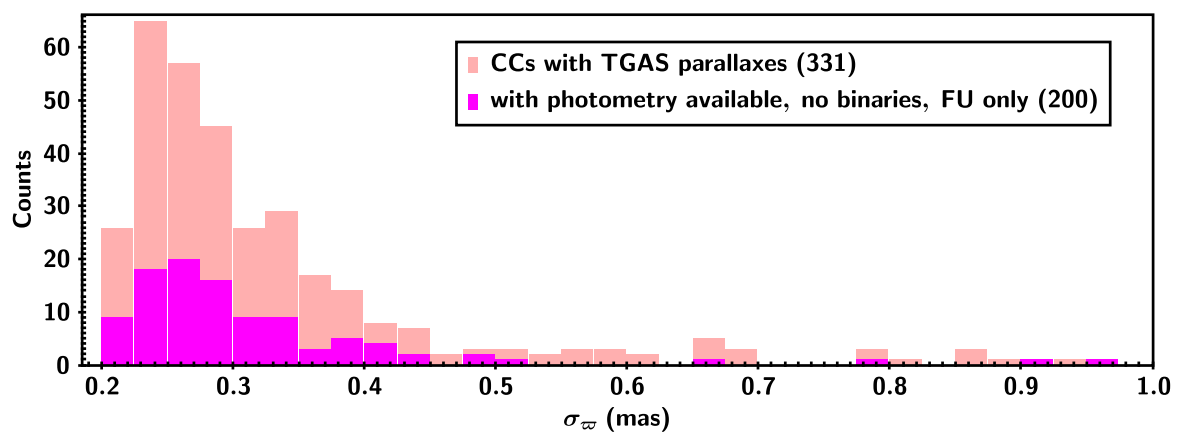

Figure 1. Error distributions of TGAS parallaxes for classical Cepheids. The pink histogram corresponds to the whole sample of 331 Galactic classical Cepheids in the TGAS catalogue, the magenta histogram shows a subsample of 102 fundamental mode classical Cepheids with $\left(V, I, J, K_{\mathrm{s}}\right)$ photometry available in the literature after removing the known binaries. The bin size is 0.025 mas.

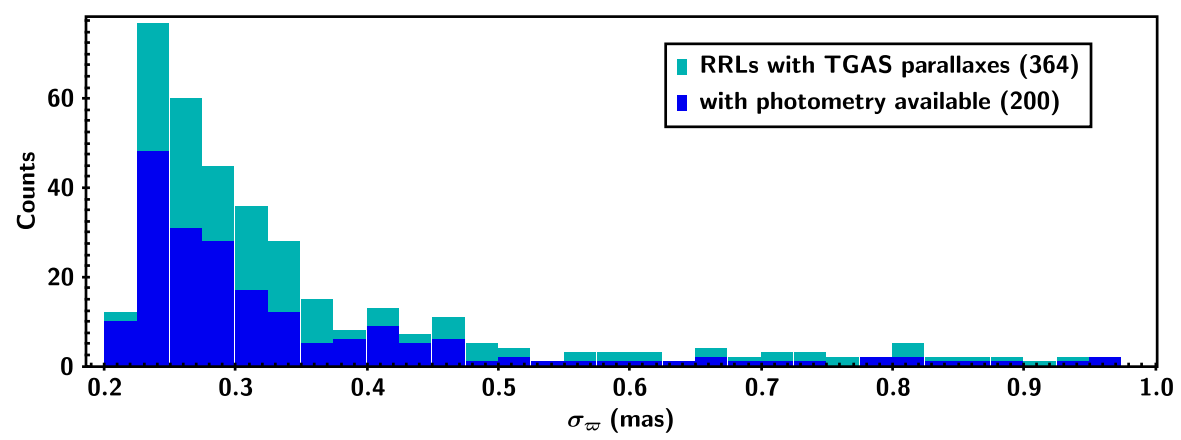

Figure 2. Error distributions of TGAS parallaxes for RR Lyrae stars. The cyan histogram corresponds to the whole sample of 364 RR Lyrae stars in the TGAS catalogue, the blue histogram shows a subsample of 200 stars with $\left(V, K_{\mathrm{s}}, W_{1}\right)$ photometry available in the literature. The bin size is 0.025 mas.

Besides $G$-band time-series photometry and pulsation characteristics for a few thousand Cepheids and RR Lyrae stars in the Large Magellanic Cloud (LMC; [8], see also Eyer et al., this volume), data for variable stars in Gaia Data Release 1 (DR1) include parallaxes for 331 classical Cepheids, 31 Type II Cepheids and 364 RR Lyrae stars, in common between Gaia and the Hipparcos and Tycho-2 catalogues. They were computed as part of the Tycho-Gaia Astrometric Solution (TGAS, [10]).

The error distributions of the TGAS parallaxes for classical Cepheids and RR Lyrae stars are shown in Figures 1 and 2, respectively. Errors range from 0.2 to about 1 milliarcsecond (mas) and peak around $\sim 0.25$ mas for both types of pulsating stars ${ }^{1}$. Although this is not comparable to the final Gaia precision, it already represents a significant general improvement with respect to Hipparcos parallaxes ([15]). Additionally, as described in [10], there could still be some systematic effects at a typical level of \pm 0.3 mas depending on the sky position and the colour of the source. However, these systematic effects are spatially correlated and become negligible for RR Lyrae stars and Type II Cepheids that are evenly distributed on the sky, while they may be relevant for classical Cepheids,

\footnotetext{
${ }^{1}$ After publication of the Gaia DR1 catalogue, a number of authors have suggested that the standard errors of TGAS parallaxes may be overestimated (e.g., [5] and Michael Feast's concluding comments, this volume).
} 


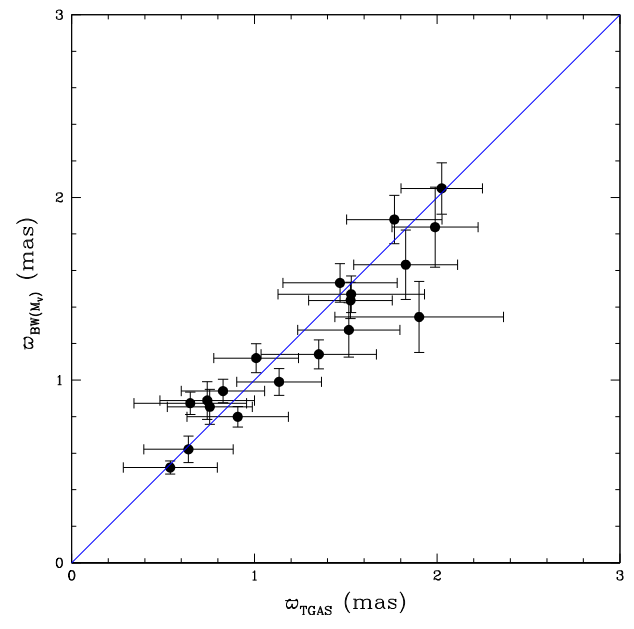

Figure 3. Comparison between the TGAS parallaxes and photometric parallaxes inferred from the absolute visual magnitude $\left(M_{V}\right)$ estimated via the Baade-Wesselink (B-W) technique for 19 Galactic RR Lyrae stars (see Table 2 in [11] and references therein). The blue line represents the bisector.

which are mainly concentrated in the Galactic disk. We also note that the TGAS samples are the result of several processing stages and truncations (e.g., removal of sources brighter than a certain limit and bluer/redder than a certain colour interval, truncation of sources with less than 5 transits, or with large astrometric uncertainties, see [7] for details), each with a different impact on the resulting sample. Nevertheless, the very limited size of the Cepheid and RR Lyrae samples and the large relative errors of TGAS parallaxes hide most of these effects.

\section{Comparison with trigonometric and photometric parallaxes in the literature}

A direct comparison between TGAS and Hipparcos parallaxes ([15]) is possible for 248 classical Cepheids, 31 Type II Cepheids and 188 RR Lyrae stars for which both measurements are available. The number of negative parallaxes is significantly reduced from $32 \%$ of the sample of classical Cepheids in Hipparcos to only $4 \%$ in TGAS, decreasing from $42 \%$ to $16 \%$ for the Type II Cepheids and from $32 \%$ to $1 \%$ for the RR Lyrae stars. This is a clear indication that uncertainties of the TGAS parallaxes are smaller than in Hipparcos. When comparing TGAS and Hipparcos parallaxes in the period-luminosity plane using the $K_{\mathrm{s}}$-band $\left(P L_{K_{\mathrm{s}}}\right)$, the improvement in quality and statistics of the former is impressive, particularly for RR Lyrae stars.

A direct comparison of TGAS versus the Hubble Space Telescope (HST) is possible for three classical Cepheids with HST parallaxes from [2],[13] and [4], one Type II Cepheid (VY Pyx) and five RR Lyrae stars with HST parallaxes from [3]. TGAS and HST parallaxes compare favourably for the classical Cepheids and even better for the RR Lyrae stars. On the other hand, the TGAS parallax of VY Pyx is much smaller than the HST value by [3], and nicely places the star on the extrapolation to longer periods of the RR Lyrae star $P L_{K}$ relation (see Fig. 10 in [7]), while this is not the case with the HST parallax.

Figure 3 shows the comparison between the TGAS parallaxes and photometric parallaxes inferred from the absolute visual magnitude $\left(M_{V}\right)$ estimated via the Baade-Wesselink (B-W) technique for 19 Galactic RR Lyrae stars (see Table 2 in [11] and references therein). The agreement is excellent both in the case of the $M_{V}$ and the $K$-band absolute magnitudes $\left(M_{K}\right)$. Similarly, good agreement is found between TGAS parallaxes and the photometric parallaxes inferred from the application of the Infrared Surface Brightness version of the B-W technique to 54 classical Cepheids from the collection 
Table 1. $P L_{K_{\mathrm{s}}}$ relations for classical Cepheids, Type II Cepheids and RR Lyrae stars with zero point based on TGAS parallaxes.

\begin{tabular}{lcc}
\hline & Relation $(\mathrm{mag})$ & r.m.s. $(\mathrm{mag})$ \\
\hline$P L_{K_{\mathrm{s}}}$ 95 objects (LSQ) & $-3.365 \log P-(2.06 \pm 0.08)$ & 0.74 \\
$P L_{K_{\mathrm{s}}} 102$ objects (ABL) & $-3.365 \log P-(2.63 \pm 0.10)$ & 0.88 \\
$P L_{K_{\mathrm{s}}} 102$ stars (BA) & $-3.365 \log P-\left(2.60_{-0.15}^{+0.11}\right)$ & 1.33 \\
\hline$P L_{K_{\mathrm{s}}} 22$ objects (LSQ) & $-2.385 \log P-(1.18 \pm 0.12)$ & 0.81 \\
$P L_{K_{\mathrm{s}}}$ 26 objects (ABL) & $-2.385 \log P-(1.58 \pm 0.17)$ & 1.10 \\
$P L_{K_{\mathrm{s}}} 26$ objects (BA) & $-2.385 \log P-\left(1.51_{-0.22}^{+0.23}\right)$ & 1.14 \\
\hline$P L_{K_{\mathrm{s}}} 195$ stars (LSQ) & $-2.73 \log P-(1.06 \pm 0.04)$ & 0.84 \\
$P L_{K_{\mathrm{s}}} 200$ stars (ABL) & $-2.73 \log P-(1.26 \pm 0.04)$ & 0.90 \\
$P L_{K_{\mathrm{s}}} 200$ stars (BA) & $-2.73 \log P-(1.24 \pm 0.05)$ & 1.02 \\
\hline
\end{tabular}

in [9]. Pulsation parallaxes for a few classical Cepheids and RR Lyrae stars in our samples have been estimated from the theoretical modelling of the stars' multi-band light curves through nonlinear convective pulsation models (Marconi, this volume) and compared with the corresponding TGAS parallaxes. Agreement is generally satisfactory except for RS Cas, a classical Cepheid for which the TGAS parallax is likely incorrect as it places the star about 2 magnitudes below the $P L$ relation.

\section{Luminosity calibrations based on different fitting approaches}

A procedure often used to calibrate the period-luminosity $(P L)$, period-Wesenheit $(P W)$, periodluminosity-metallicity $(P L Z)$ or luminosity-metallicity $\left(M_{V}-[\mathrm{Fe} / \mathrm{H}]\right)$ relations is the direct transformation to distance (hence, absolute magnitude) by parallax inversion and then the least squares fit (LSQ) of the derived parameters. However, symmetrical errors in parallaxes translate into asymmetric errors in the magnitudes, an effect that becomes specially problematic for parallaxes with large relative errors that introduces an overall bias. Furthermore, this method does not allow us to use negative parallaxes, thus biasing the samples against more distant sources. On the contrary, in methods that operate in parallax space such as the Astrometric Based Luminosity (ABL, [1]) and Bayesian approaches (hereinafter, BA), the parallaxes are used directly, thus maintaining the errors symmetrical and allowing negative parallaxes to be used. We applied all three methods (LSQ, ABL and BA) to fit the canonical relations that Cepheids and RR Lyrae stars conform to. In Table 1, we summarise the TGAS-based $P L_{K_{\mathrm{s}}}$ relations of Cepheids and RR Lyrae stars obtained with the three different fitting approaches and adopting the slope from [9] for classical Cepheids, from [14] for the Type II Cepheids and from [11] for the RR Lyrae stars. Table 1 shows that the ABL and Bayesian approaches are generally in good agreement with each other and provide brighter absolute magnitudes (hence longer distances) than the direct transformation of parallaxes and the LSQ fit. Differences are larger (of about 0.5-0.6 mag, on average) for the classical Cepheids, reduce to 0.4-0.5 mag for the Type II Cepheids, and are the smallest ones, $0.2 \mathrm{mag}$, for the RR Lyrae stars. However, we note that the r.m.s. scatter of all relations is very large, due to the large parallax uncertainties.

Figure 4 shows the results obtained for the LMC distance modulus by applying the TGAS-based relations of the RR Lyrae stars to the LMC variable stars studied by [6]. The results obtained for the RR Lyrae stars show a much better agreement among the three methods and also a reasonably good agreement with the currently adopted LMC distance modulus from [12] (solid, vertical line).

The TGAS parallaxes represent a significant improvement upon the previous Hipparcos estimates. However, the TGAS-based luminosity calibrations presented in this study have to be considered pre- 


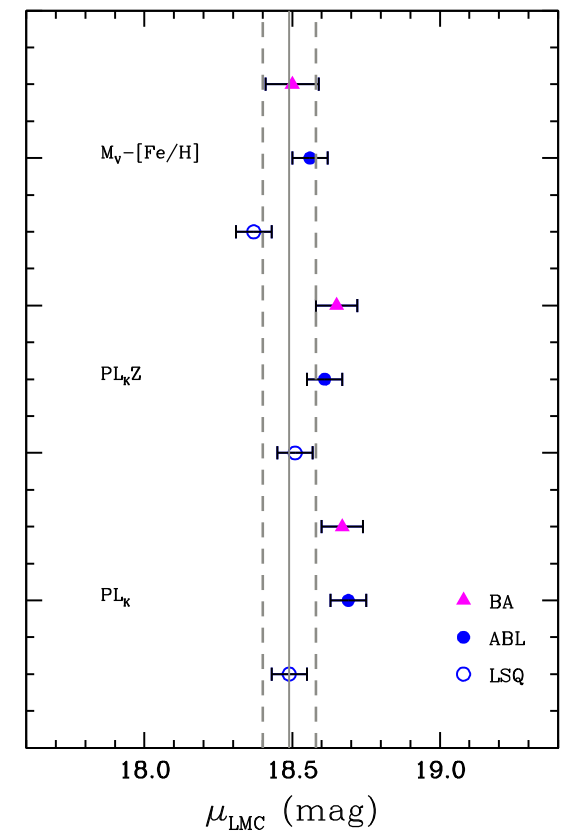

Figure 4. LMC distance moduli obtained by fitting the TGAS-based $P L_{K}, P L_{K} Z$ and $M_{V}-[\mathrm{Fe} / \mathrm{H}]$ relations of RR Lyrae stars with the LSQ (blue open circle), the ABL (blue filled circles) and the Bayesian approaches (magenta filled triangles), respectively. From bottom to top: $P L_{K_{\mathrm{s}}}$ relations for $195 / 200 / 200$ stars (LSQ, ABL, BA methods) with slope from [11]; $P L_{K_{\mathrm{s}}} Z$ relations with slope of the dependence on period from [11]; $M_{V}-[\mathrm{Fe} / \mathrm{H}]$ relations with slope from [6]. A solid vertical line shows the LMC distance modulus from [12].

liminary and to be superseded by new more accurate relations calibrated on Gaia-only parallaxes that will be published in future releases, to start with Gaia Data Release 2 in 2018.

Acknowledgments This work has made use of data from the ESA space mission Gaia, processed by the Gaia Data Processing and Analysis Consortium (DPAC), and of the SIMBAD database, operated at CDS, Strasbourg, France.

\section{References}

[1] F. Arenou, X. Luri, in Harmonizing Cosmic Distance Scales in a Post-Hipparcos Era, ed. D. Egret \& A. Heck, ASP Conference Series, 167, 13 (1999)

[2] Benedict, G. F., McArthur, B. E., Feast, M. W., et al., AJ, 133, 1810 (2007)

[3] Benedict, G. F., McArthur, B. E., Feast, M. W., et al., AJ, 142, 187 (2011)

[4] Casertano, S., Riess, A. G., Anderson, J., et al., ApJ, 825, 11 (2016)

[5] Casertano, S., Riess, A. G., Bucciarelli, B., \& Lattanzi, M. G., A\&A, 599, A67 (2017)

[6] Clementini, G., Gratton, R. G., Bragaglia, A., et al., AJ, 125, 1309 (2003)

[7] Gaia Collaboration, Clementini, G., et al., A\&A, in press (arXiv: 1705.00688) (2017)

[8] Clementini, G., Ripepi, V. Leccia, S., et al., A\&A, 595, A133 (2016)

[9] Fouqué, P., Arriagada, P., Storm, J., et al., A\&A, 476, 73 (2007)

[10] Lindegren, L., Lammers, U., Bastian, U., et al., A\&A, 595, A4 (2016)

[11] Muraveva, T., Palmer, M., Clementini, G., et al., ApJ, 807, 127 (2015)

[12] Pietrzynski, G., Graczyk, D., Gieren, W., et al., Nature, 495, 76 (2013)

[13] Riess, A. G., Casertano, S., Anderson, J., MacKenty, J., \& Filippenko, A. V., ApJ, 785, 161 (2014)

[14] Ripepi, V., Moretti, M.-I., Marconi, M., et al., MNRAS, 446, 3034 (2015)

[15] van Leeuwen, F., A\&A, 474, 653 (2007) 